\title{
Responses to holism, but no arguments against
}

\author{
Milena Ivanova: Duhem and Holism. Cambridge: Cambridge \\ University Press, 2021, 70 pp, £15.00 PB
}

\section{Corey Dethier ${ }^{1}$}

Accepted: 9 November 2021 / Published online: 15 November 2021

(c) The Author(s) 2021

The most discussed aspects of Pierre Duhem's philosophy are his claim that scientific testing is holistic and his associated instrumentalist view that the aim of science is to produce theories that are empirically adequate. Milena Ivanova's new book, Duhem and Holism, offers an introduction to both of these key tenets with a particular focus on how contemporary philosophers of science have responded to Duhem's claims about holism.

Duhem and Holism is admirably clear and engaging in its discussion of both Duhem's philosophical views and some of the many responses that they have engendered over the last century. For those unfamiliar with the material, it may serve as a worthwhile introduction, replete with connections to more contemporary or familiar debates and lively but short examples from the history of science. Experts will find something of value here as well in the discussion of the connection between what Duhem calls 'good sense' and contemporary work in social epistemology.

Unfortunately, other elements of the book are not as strong as the section on Duhem's treatment of 'good sense.' In particular, Duhem and Holism offers a surprisingly thin treatment of Duhem's actual arguments for holism. Duhem argues that - as a matter of logic_-our failure to observe the predictions of a theory does not discriminate between the various propositions employed in deriving that prediction. Duhem and Holism surveys a number of views that accept this central claim but that argue that there are resources other than those of (deductive) logic for deciding between theories-resources such as theoretical virtues, good sense, statistical tools, and inference to the best explanation.

But one might equally reject Duhem's holistic claim, an option that receives little attention. This is how Bayesian responses to Duhem should be interpreted: what philosophers like Dorling (1979) and Strevens (2001) argue is that Duhem's position is not correct because-again as a matter of logic_-some assumptions do fare better than others when a prediction fails to be observed. Similarly, philosophers

Corey Dethier

corey.dethier@gmail.com

1 Philosophy Department, Leibniz Universität Hannover, Hannover, Germany 
such as Azzouni (2000) and Morrison (2010) have argued that traditional holistic arguments like Duhem's have much less applicability than is normally thought on the ground that scientists usually only require a few specific auxiliary assumptions when deriving predictions. And one can legitimately ask whether Duhem's arguments for holism rely on a dated form of hypothetico-deductivism or whether they can be extended to domains such as biology where the instruments employed are not obviously dependent on the science in the same way that the instruments of physics are dependent on physics. Duhem and Holism does not address these questions. It is fair to say that the main question covered in the book's discussion of contemporary responses is: given the assumption that Duhem's argument succeeds, how should we respond?

Of course, a book of this size on a figure and topic as central to the history of philosophy of science as Duhem and holism is guaranteed to omit some topics that some experts might think relevant. This particular omission matters, however, due to the way that it influences the discussion of responses to holism in the last main chapter of the book. Here, Duhem and Holism does an admirable job summarizing the discussion of Darden (1991) and Mayo (1996) and raises interesting questions regarding these views, such as whether they can be said to be truly disagreeing with Duhem or, by contrast, should be viewed as 'mere' elaborations of the concept of 'good sense.' This question is important and interesting. Duhem is an explicit antiinductivist, but if (broadly) inductive strategies like those outlined by Mayo can be seen as an elaboration of 'good sense,' how should we understand his anti-inductivist commitment? Unfortunately, the connection here is only really raised in the conclusion, meaning that its interesting implications cannot be fully explored.

If the discussion of Darden (1991) and Mayo (1996) offers a detailed and evenhanded treatment that raises interesting questions without quite answering them, the discussion of the Bayesian approach is less satisfying. Duhem and Holism adopts a critical view of Bayesian responses to Duhem on the grounds that (a) Bayesianism has no satisfactory answer to the problem of the priors, (b) that it offers no specific practical advice on how to proceed when faced with an unsuccessful prediction, and (c) that Bayesianism does not offer a solution to Duhem's problem so much as a restatement of it.

As indicated above, however, these criticisms seem to miss the point of the Bayesian response. Unlike the other options surveyed, the Bayesian does not accept Duhem's argument for holism, meaning that they are not looking for practical advice about how to proceed when faced with an unsuccessful prediction. On the contrary, the Bayesian claims that Duhem is simply wrong regarding holism. As a matter of logic, an unsuccessful prediction does not have the same implications for every proposition employed in its derivation, if for no other reason than because those propositions can differ in their prior probability. 'Where do priors come from?' and 'How should I determine that a particular auxiliary assumption is false?' are good questions, but they simply are not relevant to the disagreement between Duhem and the Bayesian. As Harman (1986) has pointed out, questions about logical relations between propositions and more practical epistemic questions often come apart.

That is not to say that the Bayesian response is correct or the others wrong. There may well be good reasons to find the argument just rehearsed unsatisfactory. 
Furthermore, given that the Bayesian aims for a different kind of response to Duhem than those offered by Darden (1991) and Mayo (1996), it may be that these responses can all happily coexist. After all, these latter suggestions are designed to resolve the dilemma posed by holism on the assumption that logic is insufficient for the task - that is, they are designed to offer the kind of practical advice that the Bayesian does not. Still, the upshot is that we cannot evaluate the adequacy of the Bayesian rejoinder so long as we understand it as a response to holism rather than a rejection of Duhem's argument for the position.

Those interested in a brief introduction to either Duhem's overall philosophy or his views on 'good sense' will find Duhem and Holism to be an engaging and worthwhile read. Those interested in arguments for or against holism, however, are likely better served by looking elsewhere.

Funding Open Access funding enabled and organized by Projekt DEAL. Research funded by the Deutsche Forschungsgemeinschaft (DFG, German Research Foundation) - Project 254954344/ GRK2073/2.

Open Access This article is licensed under a Creative Commons Attribution 4.0 International License, which permits use, sharing, adaptation, distribution and reproduction in any medium or format, as long as you give appropriate credit to the original author(s) and the source, provide a link to the Creative Commons licence, and indicate if changes were made. The images or other third party material in this article are included in the article's Creative Commons licence, unless indicated otherwise in a credit line to the material. If material is not included in the article's Creative Commons licence and your intended use is not permitted by statutory regulation or exceeds the permitted use, you will need to obtain permission directly from the copyright holder. To view a copy of this licence, visit http://creativecommons.org/licen ses/by/4.0/.

\section{References}

Azzouni, Jody. 2000. Knowledge and reference in empirical science. London: Routledge.

Darden, Lindley. 1991. Theory change in science: Strategies from Mendelian genetics. Oxford: Oxford University Press.

Dorling, John. 1979. Bayesian personalism, the methodology of scientific research programmes, and duhem's problem. Studies in History and Philosophy of Science Part A 10 (3): 177-187.

Harman, Gilbert. 1986. Change in view: Principles of reasoning. Cambridge: The MIT Press.

Mayo, Deborah G. 1996. Error and the growth of experimental knowledge. Chicago: University of Chicago Press.

Morrison, Joe. 2010. Just how controversial is evidential Holism? Synthese 173 (3): 335-352.

Strevens, M. 2001. The Bayesian treatment of auxiliary hypotheses. British Journal for the Philosophy of Science 52: 515-537.

Publisher's Note Springer Nature remains neutral with regard to jurisdictional claims in published maps and institutional affiliations. 